\title{
Topographical Distribution of Consecutive Cholinergic Transmission Deficit in Patients Suspected of Dementia Disease
}

\author{
Rolf Ekedahl* \\ Department of Neurophysiology, NeuFydi Stockholm, Sweden
}

*Corresponding author: Rolf Ekedahl, Department of Neurophysiology, NeuFydi, Ångströmsgatan 7, 11269 Stockholm, Sweden, Tel: +46(0)706785783; E-mail: rolf.ekedahl@neufydi.com

Received: 14 Feb, 2020 | Accepted: 10 Mar, 2020 | Published: 17 Mar, 2020

Citation: Ekedahl R (2020) Topographical Distribution of Consecutive Cholinergic Transmission Deficit in Patients Suspected of Dementia Disease. J Neurol Neurobiol 6(1): dx.doi.org/10.16966/2379-7150.162

Copyright: (c) 2020 Ekedahl R. This is an open-access article distributed under the terms of the Creative Commons Attribution License, which permits unrestricted use, distribution, and reproduction in any medium, provided the original author and source are credited.

\begin{abstract}
Introduction: The objective of this study is to evaluate topographical differences for Acetylcholine neurotransmission deficit in suspected dementia patients measured with the electroencephalographic Vigilance-index. In order to find the most sensitive and earliest location of Acetylcholine neurotransmission deficit and find patients benefiting from Acetylcholinesterase inhibitor medication, a well-recognized treatment for suspecting Alzheimer's and Lewy body dementia.
\end{abstract}

Material and Methods: The Vigilance-index for Electroencephalography (EEG) used in the analyses: average peak power for eyes open/eyes closure reflecting Acetylcholinergic status in four locations; anterior Temporal lobe (T3 and T4), posterior Temporal lobe (T5), Parietal lobe (P3), and Occipital lobe (01) for 30 patients suspected of dementia compared to 30 healthy individuals.

The EEG recording positions according to (10-20 EEG system) covering the anterior Temporal left side and the anterior temporal right side (T4) of Cortex. The Vigilance-index average values for the positions of the suspected dementia patients compared to the control group, evaluated by Student's t-test. To illustrate differences of Vigilance-index for the recording positions, between baseline and follow-up, diagrams comparing the Vigilance-index for the recording sites constructed for the suspected dementia patients.

Results: All recording positions at the baseline showed significant increased average Vigilance-index with $p$-values $\left({ }^{* * *}\right)$ compared to the healthy controls, the highest average Vigilance-index for T3 and lowest for 01, at follow-up, the T3 increased most and 01 least. The comparison between the left and right anterior temporal regions (T3 and T4) showed different average Vigilance-index and also different Vigilance-index values for the same patient.

Conclusion: For the dementia diseases characterized by Acetylcholine Deficit: Alzheimer's disease, and Lewy body dementia, Vigilance-index can be used for early detection of these diseases. The Temporal anterior region $(T 3, T 4)$ was most and earliest detected having a cholinergic deficit and least the Occipital region (01), both at baseline and follow-up examination.

Keywords: Electroencephalography; Neurotransmitter; Dementia; Acetylcholine; Follow-up studies; Humans; Biomarkers; Nucleus basalis of Meynert; Brain Topography; Alzheimer's disease

Abbreviations: AchE: Acetylcholinesterase; AD: Alzheimer's disease; CAT: Choline acetyltransferase; CSF: Cerebrospinal Fluid; EEG: Electroencephalography; LBD: Lewy Body Dementia; NBM: Nucleus Basalis of Meynert; Vigilance-index: Quota of average EEG power at eyes open/ eyes closed

\section{Introduction}

The aim of this study is by using the Electroencephalographic (EEG) Vigilance-index to compare and locate which brain region is most sensitive and the first area affected by Acetylcholine deficit. In order to identify as early as a possible sign of dementia due to cholinergic deficit as in Alzheimer's (AD) and Lewy Body Dementia (LBD). A close relation between dementia of Alzheimer's type and the cholinergic system exists and is well-known for long, and are the logic for using Acetylcholinesterase inhibitor treatment to enhance the
Acetylcholine transmission at AD and LBD diseases [1-7]. The neurons containing Acetylcholine originate from the cell bodies located in the nucleus basalis of Meynert in the basal frontal lobe. From there, the axons spread to large areas of the cortex and via another route to the hippocampus (Figure 1). There was also a relation between the reduction of Choline Acetyltransferase (CAT), which synthesize Acetylcholine, and a correlation to intellectual impairment measured with memory tests. The muscarinic cholinergic receptor binding activity was close to what has observed in healthy brains [5], which 
therefore supports a presynaptic loss of Acetylcholine. The recently presented method to measure cholinergic status via the Vigilanceindex, a Quantitative Electroencephalographic (EEG) analysis, can identify early probable Alzheimer's and Lewy body dementia even when cognitive testing is non-pathological with Mini-mental Examination Scores (MMSE) $[8,9]$. The Vigilance-index can also be used to evaluate the effect of Acetylcholinesterase (AchE) inhibitor treatment [9] as Acetylcholinesterase inhibitor treatment hindering the breakdown of Acetylcholine and improve the postsynaptic effect by increasing the amount of available Acetylcholine. In the studies mentioned above, the measurements of Vigilance-index had been made in the Temporal, posterior region, based on the assumption that it reflects the cholinergic deficit in Alzheimer's (AD) and Lewy Body Dementia (LBD). However, there is no knowledge of the distribution of the cholinergic neurotransmission deficit in different brain regions in these diseases, except for earlier necropsy studies that have observed a selective loss of cholinergic neurons in Alzheimer's disease [1-4]. The selective loss of central cholinergic neurons overlapped with the areas that had the highest density of neurofibrillary tangles and senile plaques [1-2].

\section{Materials and Methods}

Two groups were analyzed, one group of 30 healthy persons, 17-67 years of age, and one group of 30 persons sent to a memory clinic due to suspected dementia, 68-91 years of age. The healthy persons had no complaints of memory disturbance or any self-reported alcohol or drug abuse, nor did any anticholinergic medication. The suspect dementia group neither had any reported abuse or anticholinergic/ cholinergic medication. When examined at the memory clinic with EEG, they were selected due to pathological Vigilance-index at baseline that increased at follow-up examination representing primary dementia with an increasing cholinergic deficit. The follow-up time varied and was due to the need to re-examine the patient for more definitive dementia diagnose.

EEGs recorded on awake patients, sitting on ordinary chairs, and instructed to open eyes for 30 seconds and close eyes for 90 seconds. Four of these EEG epochs with eyes closed and with eyes open analyzed and an average value calculated for respective eyes opened and eyes closed epochs.

This procedure ensured alertness and vigilance of the patients, which also monitored by the EEG assistant during the recording session. A few patients and parts of the EEG recordings that showed signs of drowsiness, and patients who were very tense or nervous with low EEG amplitudes during eye-closure, comparable to magnitudes at eye-opening [10], were excluded from the analyses.

EEGs recorded and analyzed using standard digital EEG equipment (Nervus ${ }^{\mathrm{mw}}$ 5.3 digital EEG system, Viasys Healthcare, Inc., San Diego, California, United States). The recorded analog signal with a reference electrode located between $\mathrm{Pz}$ and $\mathrm{Cz}$ (10-20 EEG system) converted to a digital signal by Fast Fourier transformation (FFT) with a sampling frequency of $128 \mathrm{~Hz}$. At least four EEG epochs, of 90 seconds with eyes closed and 30 seconds with eyes opened recorded, and approximately eighty seconds of each eye closed epoch and twenty-five seconds with eyes open used in the analysis, rarely slightly shorter epochs analyzed due to recording interference. The first 10 seconds were excluded from eyes closed epochs to avoid analyses of arousal and movement artifacts, and the first 3 seconds of eyes open epochs similarly excluded. The average peak power for four eye closure epochs and open eye epochs, within $\pm 1 \mathrm{~Hz}$ range of average peak-frequency with eyes closed, calculated. Vigilance-index decided from the ratio of the average peak

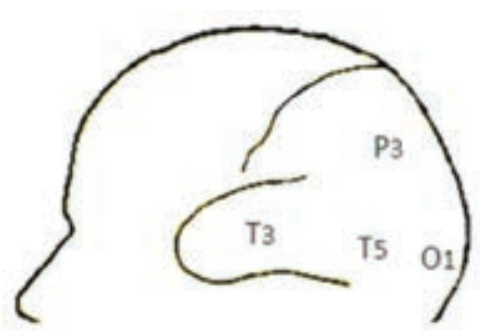

Cholinergic pathways innervating the Brain

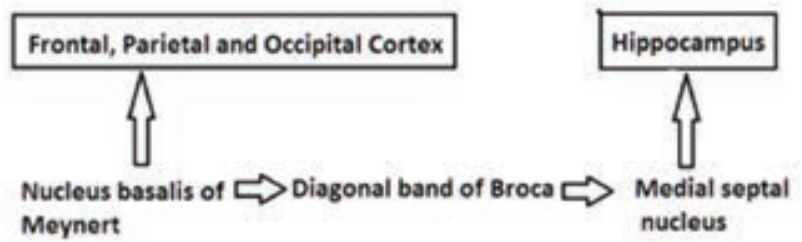

Figure 1: The EEG recording positions and the principal Cholinergic pathways innervating the Brain.

The Vigilance-index measured at the T3 position, temporal anterior lobe. T5, the Temporal, posterior lobe. P3 reflecting the parietal region and 01 the Occipital area. All these on the left side of the Brain illustrated on the top. Below the cholinergic pathways. The neurotransmitter acetylcholine's cell bodies located in Nucleus basalis frontalis (Nucleus of Meynert), the basal frontal brain area anterior to the third ventricle. The axons then spread in two different pathways, one directly to widespread cortical areas, the other one to the Hippocampus via the diagonal band of Broca and medial septal nucleus.

power for open eyes epochs and closed eyes epochs (E.O. power/E. Cl. power) and quantified relative desynchronization of average peak power. The peak-frequency and power measurements defined with the EEG filter setting of 3-15 Hz, which covered alpha and theta frequency bands $(4-13 \mathrm{~Hz})$ in order to avoided analysis of eye movement and muscle artifacts.

EEGs analyzed from the T3, T4, T6, P3 and O1 recording sites (10-20 EEG system) (Figure 1) which reflects the parts of the brain, known to be affected by Alzheimer's and Lewy body dementia and also the parts of the cortex were basic rhythm is prominent, which is needed to decide Vigilance-index. The average value for Vigilanceindex calculated and the statistical significance of the deviation for the different recording positions at baseline and follow-up examination compared to the healthy control group and p-values calculated with two-tails distribution and different variance for the Student's t-test $\mathrm{p}$-values. For the average Vigilance-index comparison between baseline and follow-up p-values calculated with two tails paired Student's t-test (Table 1). The Pearson product-moment correlation coefficient used comparing the correlation between the anterior Temporal recording position on each side. Also, the number of patients with the same, increased, or decreased Vigilance-index when T3 compared with T4 was calculated (Figure 2).

\section{Results}

When the patients with suspected dementia were evaluated both at the baseline, and follow-up examination, the mean-values 


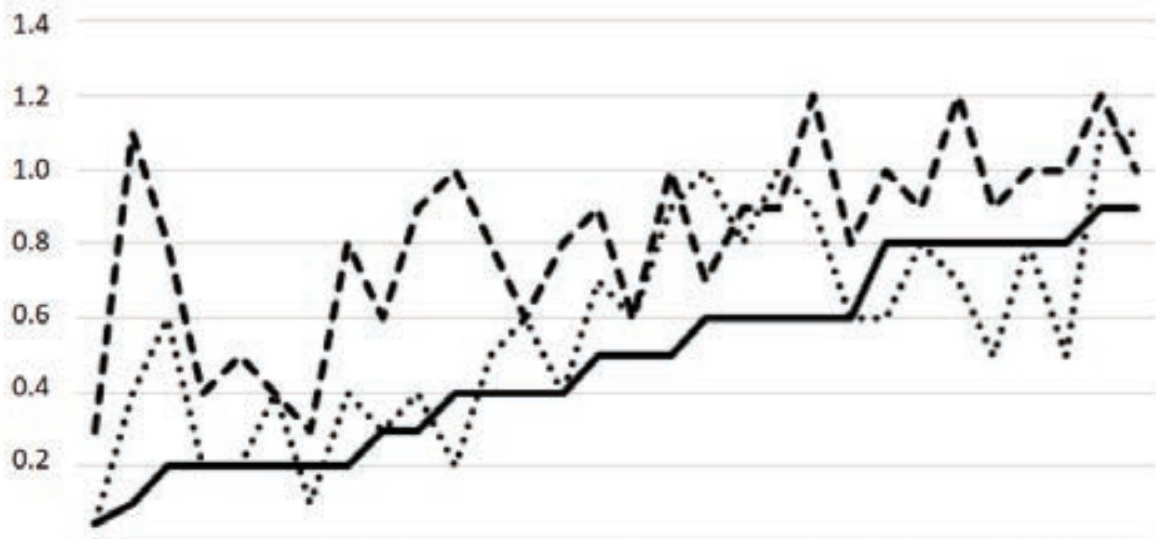

123456789101112131415161718192021222324252627282930

Figure 2: The different Vigilance-index of the T3 at baseline and follow-up and comparison with the T4 recording position at baseline.

The baseline Vigilance-index values (X-axis) for the examined patient arranged according to their values (filled line). The dashed line shows the follow-up values for the respective patient. The dotted line T4 Vigilance-values compared to the T3 values of the same patient.

Table 1: Statistical analysis by p-values of average Vigilance-index for the different recording positions and comparisons.

\begin{tabular}{|c|c|c|c|c|}
\hline $\begin{array}{c}\text { Student's t-test pvalue } \\
\mathbf{p}<0.05=* \\
\mathbf{p}<0.01=* * \\
\mathbf{p}<0.001=* * *\end{array}$ & T3 & T5 & P3 & 01 \\
\hline Healthy controls-baseline values & $* * *\left(4.5 \times 10^{-8}\right)$ & $* * *\left(3.1 \times 10^{-7}\right)$ & $* * *\left(7.0 \times 10^{-7}\right)$ & $* * *\left(8.0 \times 10^{-6}\right)$ \\
\hline Healthy controls follow-up values & $* * *\left(1.7 \times 10^{-15}\right)$ & $* * *\left(2.4 \times 10^{-13}\right)$ & $* * *\left(1.7 \times 10^{-10}\right)$ & $* * *\left(2.8 \times 10^{-8}\right)$ \\
\hline Baseline Follow-up values & $* * *\left(2.5 \times 10^{-9}\right)$ & $* * *\left(1.9 \times 10^{-4}\right)$ & $* * *\left(7.3 \times 10^{-5}\right)$ & $* *\left(1.0 \times 10^{-2}\right)$ \\
\hline
\end{tabular}

The different recording positions in the upper row and the comparisons in the left column. The p-values for the healthy controls, when baseline and follow-up values compared, were analyzed with two-tails distribution and different variance for the p-values. In the lowest row, when the baseline average Vigilance-index compared with the follow-up values, two tailed distribution, and paired p-values calculated.

for Vigilance-index was statistically significant increased $\left(^{* * *}\right)$ in all recording positions compared to the mean value for the healthy controls (Table 1), which supported that the studied patients had dementia with a cholinergic deficit and widespread distribution. The highest increase of mean Vigilance-index at baseline was for the T3 recording position and lowest for $\mathrm{O} 1$, and in-between for T5 and P3 (Figure 3). The relative increase of Vigilance-index between the baseline and follow up was also highest for T3 with statistical significance deviation $\left(^{* *}\right)$ and least for $\mathrm{O} 1\left(^{* *}\right)$ and in-between for T5 and P3 (***) (Figure 2). These distributions of cholinergic deficit show a general trend of increase of Vigilance-index for all recording positions with the highest cholinergic deficit in the temporal anterior region and lowest deficit in the Occipital region. When analyzing individual patients, the cholinergic deficit revealed a more heterogenic distribution of deficits than the described general trend, in some patients was the highest deficit in other recording positions than in the Temporal anterior region (T3) most often the neighboring (T5, P3), representing posterior Temporal and Parietal region. The above-described discrepancies between recording positions showing different deficits, could be demonstrated by a comparison between the left side with the right side anterior Temporal recording site, $8 / 30$ had the same Vigilance-index on both sides, 16/30 had higher Vigilance-index on the right side, and 6/30 had lower (Figure 3). Also, the average Vigilance-index value was different between the sides, 0.58 (T4) on the right side and 0.49 (T3) on the left side, when analyzing the differences with Pearson product-moment correlation coefficient between the T3 and T4 recording positions, the quota was 0.74 . These data clearly show the cholinergic deficit differences between rights and left anterior Temporal recording sites, and support the results that the cholinergic deficit varies between different recording locations and patients. Even though, the general trend is that the most affected area of cholinergic deficit in early dementia is the anterior temporal region and least the occipital region.

\section{Discussion}

There is a close relation between dementia and the cholinergic system with a cholinergic deficit for Alzheimer's and Lewy body dementia [1-7]. Therefore, Acetylcholinesterase inhibitor treatment hindering the breakdown of acetylcholine is a well-established treatment for these dementia diseases. Pharmacological studies of cholinergic and anticholinergic substances and AChE inhibitor medication on EEG [9,11-24]. Show that the anticholinergic substances lead to insufficient blocking of EEG amplitude when the eyes are open in healthy subjects. A comparison between healthy subjects and patients with suspected $\mathrm{AD}$ revealed significantly reduced desynchronization reaction at eye-opening of EEG in patients with suspected $\mathrm{AD}[8,9,18]$. The 


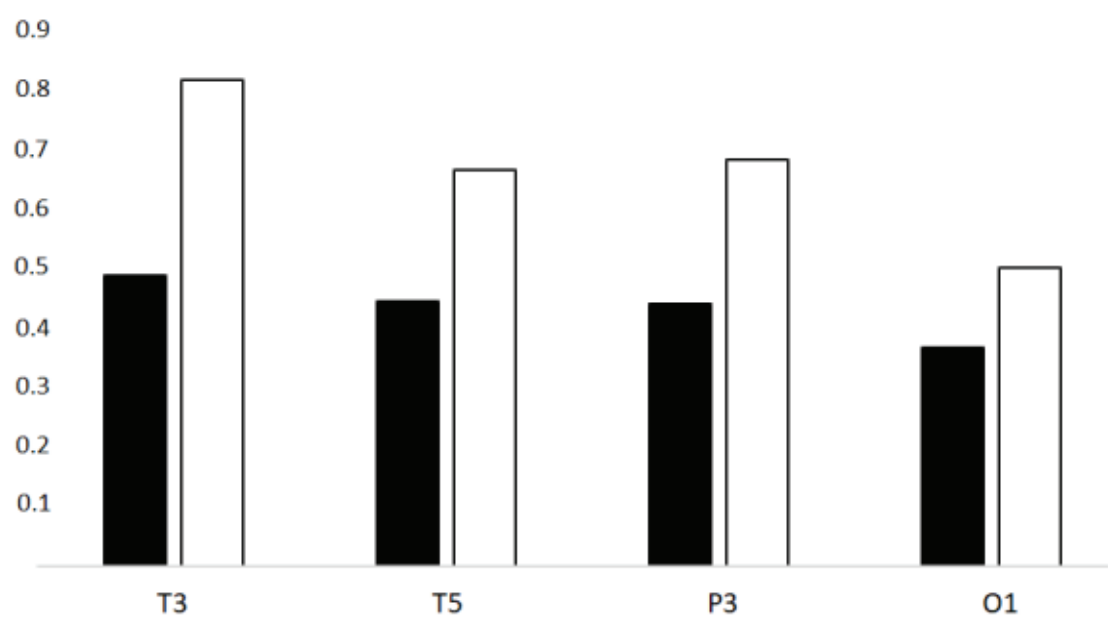

Figure 3: The Vigilance-index decided for baseline and follow-up examinations.

The baseline values filled bars (black), and the follow-up values unfilled bars. The Y-axis, Vigilance-index and the X-axis the different recording positions.

Vigilance-index constructed from these observations to measure and decide the neurotransmitter Acetylcholine function on EEG and evaluate Acetylcholinesterase inhibitor treatment and identify probable Alzheimer's and Lewy body dementia early in these disease's development $[8,9]$. Other attempts to measure the Acetylcholine function by EEG has recently presented estimating the cholinergic status in patients with dementia diseases, these methods based on algorithms of analyzing EEG-signals from several electrodes and different aspects of electrical changes in EEG [25-27]. The use of several electrodes and variables increases the possible sources of error, which perhaps might restrict the use of these methods. The Vigilanceindex measures the electrodes individually and only one variable based on peak power with eyes open and closed, which makes it possible to study the topographical distribution of cholinergic deficit. To use a quota of average EEG power for eyes open/eyes closed erases power differences between individuals and makes comparison easier between patients and comparisons on different occasions for a patient.

One recent study, when patients examined for suspected dementia at baseline and follow-up after approximately two years, the evaluation revealed that Vigilance-index best-identified patients at baseline, which developed cognitive decline by MMSE at the follow-up examination. Better, compared to biomarker components: Amyloid $\beta^{-42}$, total-Tau, and phospho-Tau in Cerebrospinal fluid (Csf). Some of these patients had a pathological Vigilance-index even when they were cognitively normal, according to MMSE, but developed cognitive decline (MMSE) at follow-up [8]. In another study comparing which EEG variable that best identified suspected dementia patients from healthy controls; the best variable was Vigilance-index [9]. In the same study, when differences in cholinergic deficit, indicated with the Vigilance-index, compared AchE inhibitor-treated patients with untreated, the baseline average Vigilance-index was unchanged for the treated group at follow-up after approximately one year. The untreated patients had significantly increased Vigilance-index at follow-up, which could be expected of a progressive decline for the Acetylcholine transmission in dementia disease with cholinergic deficits $[8,9]$. In these studies, comparisons made between a baseline and a followup examination recorded from the posterior Temporal lobe (EEG position T5, T6) either on the right or left side [8,9]. Even though the recordings from the Temporal, posterior region identified patients that had pathological Vigilance-index when normal cognition measured with MMSE [8], this study identifies the temporal anterior region on average as the most sensitive for identifying pathological cholinergic neurotransmission deficits and the occipital region as the least sensitive.

When measuring cholinergic status with Vigilance-index, it will be restricted to the areas that have relative prominent basic rhythm in the Alfa and Theta EEG frequencies band $(4-13 \mathrm{~Hz})$, which is the post central part of the brain containing Temporal, Parietal and Occipital regions [10]. Due to this circumstance, the Frontal lobe's Vigilanceindex cannot generally be directly examined by surface EEG. However, the Cholinergic axons originate from the Cholinergic cell bodies in the nucleus basalis of Meynert, situated in the basal frontal lobe, from there the axons spread to large areas of the cortex and via another route to the hippocampus (Figure 1) $[1,5,28]$. Except for the Frontal lobe, the temporal anterior region is probably the cortex region which is closest to the nucleus basalis of Meynert in the Frontal lobe. Therefore, the EEG recorded from the cortex surface of the temporal anterior region may reflect the cholinergic deficit from the frontal lobe.

Based on this assumption, on average, the earliest and most pronounced cholinergic deficit occurs in the basal frontal region measured with Vigilance-index from the temporal anterior region (T3, T4). This conclusion is also well consistent with the observed selective destruction of cholinergic cell bodies in the nucleus basalis of Meynert and the first detected intraneural neurofibrillary changes in the basal frontal region in Alzheimer's dementia [1,28,29].

In general, the cholinergic deficit measured with Vigilance-index influenced the Temporal anterior region most and then the Parietal region and Temporal, posterior region, and least Occipital region, when baseline examination compared to follow-up observations (Figure 2). However, when looking at individual patients, there were some variations in the recording site most influenced by the cholinergic deficit, both at the baseline and follow-up examination. These differences in Vigilance indices and the most impact of the recording site were most affected by the cholinergic deficit that was described between patients when comparing the left and right sides of the temporal anterior region (Figure 3). Therefore, a varying influence 
of cholinergic deficit at different cortex areas on the top of the average influence observed in this study, which probably can explain some of the differences in the symptoms between patients and the progression of dementia with cholinergic deficit such in Alzheimer's disease and Lewy body dementia.

In clinical practice, when Csf diagnostics to measure Amyloid $\beta^{-42}$, total-Tau, and phospho-Tau to identify dementia diseases, the spatial distribution of the disease cannot be estimated. Instead, the spatial distribution can be used for early diagnostics when the Vigilanceindex used for diagnostics of dementia, as the Vigilance-index can measure EEG activity originating from the basal frontal lobe, which reflects the most sensitive site to measure cholinergic deficit in general, but also from other cholinergic deficit sensitive sites. Supported by a study of patients with suspected dementia examined with MMSE, Vigilance-index, and the commonly used Csf biomarkers: Amyloid $\beta^{-42}$, total-Tau, and phospho-Tau. The most sensitive variable to predict the number of patients with cognitive decline after approximately two years was the Vigilance-index, after that total-Tau, Amyloid $\beta^{-42}$, and least sensitive phospho-Tau in Csf [8], the recorded EEG activity from the posterior part of the Temporal lobe (T5, T6). This study has shown the Temporal, posterior region (T5, T6 EEG sites) not to be the most sensitive recording site in general for the cholinergic deficit. Instead, the most sensitive is the anterior Temporal recording site (T3, T4 EEG sites) demonstrated in the current study.

An advantage with the topographic recording of Vigilance-index, it is easy to identify the most sensitive site for the cholinergic deficit and choose that for analyses. Besides, the EEG recording and analyses are inexpensive, and a method easily accessible for the patient to cooperate, without the restriction of anticoagulant treatment.

\section{Conclusion}

When the Vigilance-index used to explore different sites of the cortex to identify cholinergic deficit, the average value showed that the Temporal anterior region to be most sensitive for identifying cholinergic deficit by increased Vigilance-index and the least the occipital cortex, early in the dementia disease. The Temporal anterior cortex reflects most probably the cholinergic axons to the cortex and originating in the nucleus basalis of Meynert in the basal frontal lobe and the origin of the cholinergic neurons cell bodies known to be affected in the dementia process. However, there was some variation in which of the recording site most influenced by the cholinergic deficit and increased Vigilance-index, which could be identified by the topographic approach. The clinical value of this topographical EEG analysis method is that it leads to a simple method for early identification of dementia disease, which probably enhances therapeutic measures as they can be instituted early in the dementia disease.

\section{Acknowledgments}

The author thanks Gunilla Brohme' at Nacka hospital for valuable help getting access to data.

\section{Funding sources}

This research not funded by any specific grant from funding agencies in the public, commercial, or not-for-profit sectors.

\section{Conflict of Interests}

None.

\section{References}

1. Davies P, Maloney A (1976) Selective loss of central cholinergic neurons in Alzheimer's disease. Lancet 308: 1403.

2. Perry EK, Tomlinson BE, Blessed G, Bergmann K, Gibson PH, et al. (1978) Correlation of cholinergic abnormalities with senile plaques and mental test scores in senile dementia. Br Med J 2: 1457-1459.

3. Perry EK, Perry RH, Blessed G, Tomlinson BE (1977) Necropsy evidence of central cholinergic deficits in senile dementia. Lancet 1: 189.

4. Francis PT, Palmer AM, Sims NR, Bowen DM, Davison AN, et al. (1985) Neurochemical studies of early-onset Alzheimer's disease. Possible influence on treatment. N Engl J Med 313: 7-11.

5. Coyle JT, Price DL, DeLong MR (1983) Alzheimer's disease: a disorder of cortical cholinergic innervation. Science 219: 1184-1190.

6. Drachman DA, Leavitt J (1974) Human memory and the Cholinergic system. Arch Neurol 30: 113-121.

7. Perry EK, Blessed G, Tomlinson BE, Perry RH, Crow TJ, et al. (1981) Neurochemical activities in human temporal lobe related to aging and Alzheimer-type changes. Neurobiol Aging 2: 252-256.

8. Ekedahl R (2019) Comparison of Mini Mental Score Examination, Quantitative Electroencephalography and Cerebrospinal Fluid Biomarkers in Clinical Practice Examining Dementia. Int J Psychol Behav Anal 5: 1-5.

9. Ekedahl R (2019) Comparison of cholinergic status with quantitative EEG parameters in healthy subjects and patients suspected of dementia. Arch Biomed Eng \& Biotechnol 1: 1-6.

10. Niedermeyer E (2007) The normal EEG of the waking adult. In: Niedermeyer E, Silva FLd (eds) Electroencephalography. $5^{\text {th }}$ edition, Lippincott, Williams and Wilkins, Philadelphia, USA 167-192.

11. Agnoli A, Martucci N, Manna V, Conti L, Fioravanti M (1983) Effect of Cholinergic and anticholinergic drugs on short term memory in Alzheimer's dementia: a computerized Electroencephalographic study. Clin Neuropharmacol 6: 311-323.

12. Sannita WG, Maggi L, Rosadini G (1987) Effects of Scopolamine (0.25$0.75 \mathrm{mg}$ i.m.) on the Quantitative EEG and the Neuropsychological status of healthy volunteers. Neuropsychobiology 17: 199-205.

13. Sloan EP, Fenton GW, Standage KP (1992) Anticholinergic drug effects on quantitative Electroencephalogram, visual evoked potentials and verbal memory. Biol Psychiatry 31: 600-606.

14. Neufeld MY, Rabey MJ, Parmet Y, Sifris P, Treves TA, et al. (1994) Effects of single intravenous dose of scopolamine on the quantitative EEG in Alzheimer's disease patients and age-matched controls. Electroencephalogr Clin Neurophysiol 91: 407-412.

15. White RP, Rinaldi F, Himmich HE (1956) Central and peripheral nervous effect of Atropine sulfate and Mepiperphenidol bromide (Darstine) on human subjects. J Appl Physiol 8: 635-642.

16. Martin WR, Eades CG (1960) A Comparative Study of the Effect of Drugs on Activating and Vasomotor Responses Evoked by Mid Brain Stimulation: Atropine, Pentobarbital, Chlorpromazine and Chlor promazine Sulfoxide. Psychopharmacologia 1: 303-335.

17. Longo VG (1966) Behavioral and Electroencephalographic effects of Atropine and related compounds. Pharmacol Rev 18: 971-974.

18. van der Hiele $\mathrm{K}$, Bollen EL, Vein AA, Reijntjes RH, Westendorp RG, et al. (2008) EEG markers of future cognitive performance in the elderly. J Clin Neurophysiol 25: 83-89. 
19. Alhainen K, Partanen J, Reiniainen K, Laulumaa V, Soininen $\mathrm{H}$, et al. (1991) Discrimination of Tetrahydoaminoacridine responders by a single dose pharmaco-EEG in patients with Alzheimer's disease. Neurosci Lett 127: 113-116.

20. Reeves RR, Struve FA, Patrick G (2002) The Effects of Donepezil on Quantitative EEG in Patients with Alzheimer's disease. Clin Electroencephalogr 33: 93-96.

21. Holl G, Straschill M, Thomsen T, Fischer JP, Kewitz H (1992) Effect of the cholinesterase inhibiting substance Galanthamine on Human EEG and visual evoked potentials. Electroencephalogr Clin Neurophysiol 82: 445-452.

22. Babiloni C, Casseta E, Dal Forno G, Del Percio C, Ferreri F, et al. (2006) Donepezil effects on sources of cortical rhythms in mild Alzheimer's disease: Responders vs. Non-Responders. Neuroimage 31: 1650-1665.

23. Babiloni C, Del Percio C, Bordet R, Bourriez JL, Bentivoglio M, et al (2013) Effects of Acetylcholinesterase inhibitors and Memantine on resting-state electroencephalographic rhythms in Alzheimer's disease patients. Clin Neurophysiol 124: 837-850.

24. Davidsson P, Blennow K, Andreasen N, Eriksson B, Minthon $L$, et al. (2001) Differential increase in cerebrospinal fluid- acetylcholinesterase after treatment with acetylcholinesterase inhibitors in patients with Alzheimer's disease. Neurosci Lett 300: 157-160.

25. Babiloni C, Frisoni GB, Vecchio F, Pievani M, Geroldi C, et al. (2010) Global Functional Coupling of resting EEG Rhythms is Related to White-Matter Lesions Along the Cholinergic Tracts in Subjects with Amnesic Mild Cognitive Impairment. J Alzheimers Dis 19: 859-871.

26. Snaedal J, Johannesson GH, Gudmundsson TE, Blin NP, Emilsdottir AL, et al. (2012) Diagnostic Accuracy of Statistical Pattern Recognition of Electroencephalogram Registration in Evaluation of Cognitive Impairment and Dementia. Dement Geriatr Cogn Disord 34: 51-60.

27. Johannsson M,SnaedalJ, Johannesson GH, Gudmundsson TE, Johnsen K (2015) The Acetylcholine Index: An Electroencephalographic Marker of Cholinergic Activity in the Living Human Brain Applied to Alzheimer's Disease and Other Dementias. Dement Geriatr Cogn Disord 39: 132-142.

28. Whitehouse PJ, Price DL, Struble RG, Clark AW, Coyle JT, et al. (1982) Alzheimer's disease and senile dementia: loss of neurons in the basal forebrain. Science 215: 1237-1239.

29. Braak H, Braak E (1991) Neuropathological stageing of Alzheimerrelated changes. Acta Neuropathol 82: 239-259. 